\section{Modernes Management der Operationsabteilung am Kantonsspital Winterthur}

\author{
P. Wigger
}

Zunehmender Kostendruck und Mangel an ausgebildetem Instrumentierpersonal haben uns in den letzten Jahren gezwungen, den Operationsbetrieb zu rationalisieren. Ziel war es, durch optimale Nutzung der Ressourcen einen möglichst effizienten und qualitativ hochstehenden Operationsbetrieb zu gewährleisten. Von Anfang an war klar, dass dies in einem Betrieb, wo traditionsgemäss die Hierarchie den Ablauf bestimmt, nur durch einschneidende Änderung in der Organisationsstruktur möglich sein wird: Die Operationssäle wurden aus dem direkten Einflussbereich der Klinikleiter herausgelöst und in den Verantwortungsbereich des OPS-Managers übergeben. Für diese Aufgabe wurde ein Leitender Arzt der chirurgischen Klinik bestimmt, der bereits im Betrieb integriert war. Weitere wichtige Massnahmen waren die Unterstellung des gesamten Pflegepersonals im Operationsbereich unter eine einheitliche Leitung, die zentrale EDV-gestützte Anmeldung der Operationen sowie ein Controlling. Mit den getroffenen Massnahmen wurde innerhalb von zwei Jahren eine optimale Ausnutzung der Ressourcen erreicht. Allerdings wurde durch diese Optimierungsmassnahmen der Druck auf das Personal deutlich erhöht. Wir haben diese (negative) Auswirkung ernst genommen und einen Teil der Einsparungen in das Projekt "TOPS» investiert mit dem Ziel, die Qualität des Arbeitsplatzes und die Zufriedenheit des Personals zu verbessern.

\section{Ausgangslage: Limitierte Ressourcen \\ Bis vor wenigen Jahren war der Betrieb der Opera- tionsabteilung an einem Kantonsspital relativ unpro- blematisch. Jede Klinik hatte fix zugeteilte Opera- tionssäle. Die so definierte Operationskapazität lag meist deutlich höher als der tatsächliche Bedarf. Ope- rationssäle waren nicht selten dezentral gelegen - möglichst nahe im Bereich der Klinik, welche diesen}

Korrespondenz:

Dr. med. P. Wigger

Leitender Arzt Gefässchirurgie und OPS-Manager

Kantonsspital Winterthur

CH-8400 Winterthur
Saal benutzte. Die Operationsprogramme wurden dezentral von den Kliniken erstellt. Bei geringem Operationsvolumen standen diese Säle dann nicht selten bereits ab Mittag leer. Da keine Absprachen stattfanden und diese Säle so quasi der entsprechenden Klinik gehörten, konnte diese Kapazität nicht von anderen genutzt werden. Da ein Controlling fehlte und die Kosten offenbar keine entscheidende Rolle spielten, wurde dieser Zustand von allen Beteiligten geschätzt, da er den operativen Kliniken einen grossen Handlungsspielraum ermöglichte. Auf der anderen Seite aber führte diese mangelnde Saalauslastung (die durchschnittliche Auslastung der elektiven Operationssaalkapazität am KSW lag 1998 bei nur 68\%!) zu einer schlechten Ausnutzung der zur Verfügung stehenden Ressourcen und damit $\mathrm{zu}$ unnötigen Kosten.

Dieser Zustand wurde in den letzten Jahren durch zwei Faktoren in Frage gestellt:

- der zunehmende Kostendruck;

- der Mangel an ausgebildetem Instrumentierpersonal (Operationsschwestern und Technische Operationsassistenten und -assistentinnen).

Durch die massive Kürzung der Kantonsbeiträge ans Kantonsspital Winterthur von 60 Mio. Fr. im Jahre 1998 auf noch 45 Mio. Fr. im Jahre 2000 waren wir gefordert, alle möglichen Rationalisierungsmassnahmen auszuschöpfen. Eine auswärtige Betriebsanalyse zeigte unter anderem im Operationsbereich ein grosses Potential zur Effizienzsteigerung auf.

\section{Ziel}

Ziel war es, durch die optimale Nutzung der vorhandenen limitierten Ressourcen einen effizienten und qualitativ hochstehenden Operationsbetrieb zu gewährleisten.

\section{Lösungsansatz}

Es war klar, dass dieses Ziel nur durch eine einschneidende Änderung der Organisationsstruktur der gesamten Operationsabteilung möglich sein würde. Die Umstrukturierung beinhaltete folgende Neuerungen (vgl. auch Organigramm):

- Führung der Operationsabteilung durch die neu geschaffene Funktion des OPS-Managers und damit Herauslösen der Operationssäle aus dem direkten Verantwortungsbereich der Kliniken (Chefärzte);

- Unterstellung des gesamten Pflegepersonals im Operationsbereich unter eine einheitliche Leitung;

- zentrale EDV-gestützte Anmeldung der Operationen;

- Controlling;

- Anpassen der zugeteilten Operationskapazität gemäss der durch das Controlling ausgewiesenen Auslastung. 


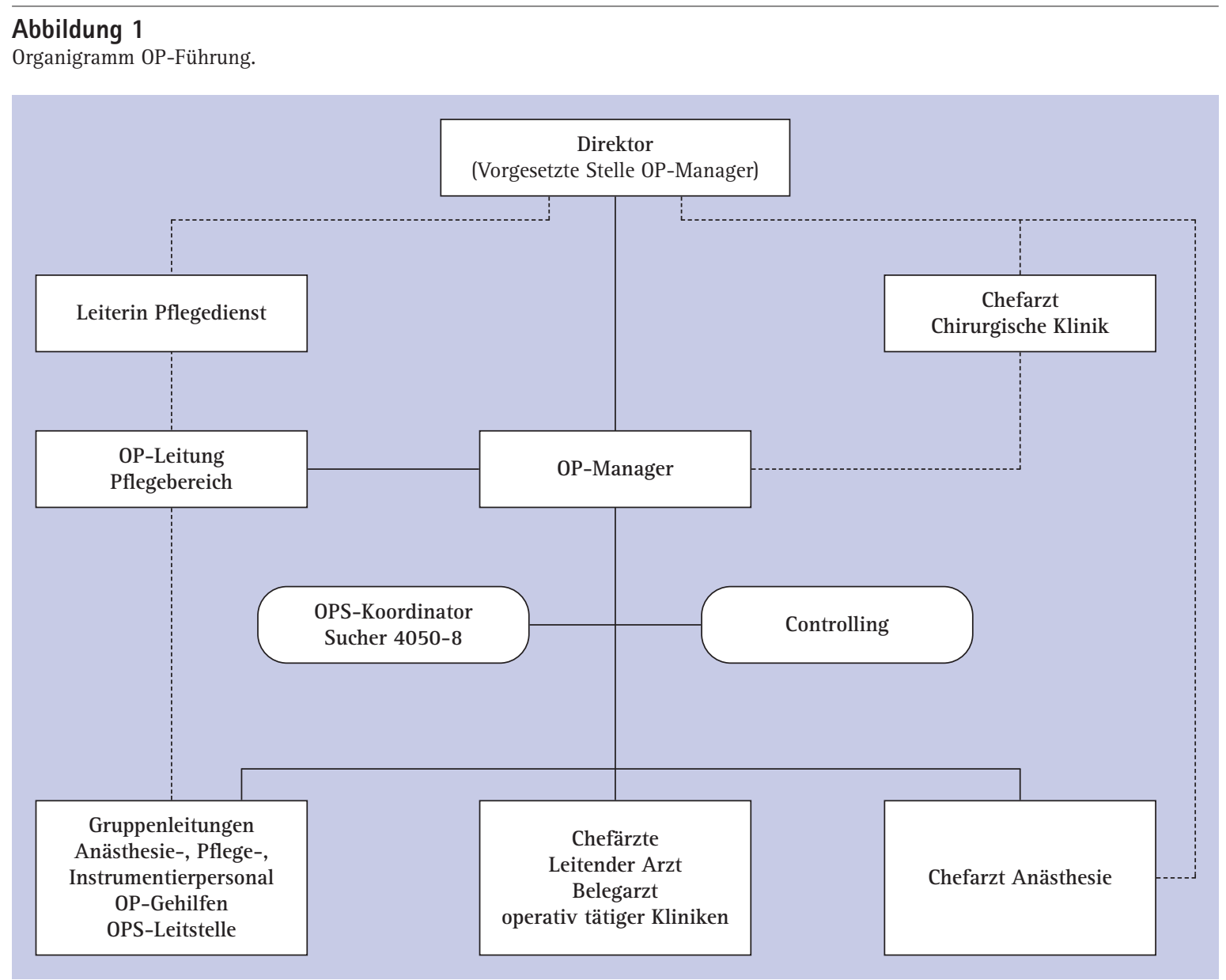

\section{OPS-Manager}

Um die Operationssäle effizient bewirtschaften zu können, wurden diese aus dem direkten Einflussbereich der Klinikleiter herausgelöst und in den Verantwortungsbereich des OPS-Managers übergeben. Den Kliniken wurde - wie bisher - eine fixe OPS-Kapazität zugeteilt. Diese wurde aber gemäss ausgewiesener Kapazitätsnutzung der Klinik teilweise deutlich reduziert. Alle Operationen müssen bis am Vortag vor $15.00 \mathrm{Uhr} z e n t r a l$ angemeldet werden. Nicht genutzte Kapazität einer Klinik verfällt zu diesem Zeitpunkt und kann vom OPS-Manager einer andern Klinik zugeteilt oder angeboten werden.

Der OPS-Manager ist auch zuständig für die rechtzeitige Bekanntgabe von voraussehbaren Saalschliessungen z. B. bei Personalmangel. Er informiert die Kliniken über allfällige Saalreduktionen und entscheidet in diesem Fall, wer wann operieren kann. Dabei arbeitet er eng mit dem Leiter des Pflegepersonals (Anästhesie-, Instrumentier- Lagerungs- und Hilfspersonal) im OPS zusammen. Die Tatsache, dass er dabei nur noch einen Ansprechpartner für das gesamte Pflegepersonal im OPS hat und nicht mehrere wie früher, erleichtert diese Aufgabe wesentlich. Der OPS-Manager ist dem Direktor des Spitals unterstellt. Vgl. Organigramm (Abb. 1).

\section{Zentrale EDV-gestützte Anmeldung von Operationen}

Die Anmeldung der Operationen erfolgt zentral via Mail, Fax oder Hauspost. Sie kann beliebig im voraus gemacht werden und ermöglicht zusammen mit einer zentralen Erfassung der Absenzen von Operateuren eine optimale Planung. Das EDV-Programm ist gekoppelt mit der Personaleinsatzplanung der Ärzte, so dass allfällige Abwesenheiten oder versehentliche Doppelbelegungen vom System erkannt werden.

\section{OPS-Koordinator: Disponent für Tagesablauf}

Im lebhaften Operationsbetrieb eines öffentlichen Spitals sind Notfalloperationen häufig. Diese können vom Operateur dem OPS-Koordinator telefonisch angemeldet werden. Für diese Funktion wurden einige erfahrene Personen aus dem Pflegebereich ausgebildet. Dieser informiert die Anästhesie sowie das Pflegepersonal im OPS und plant die zur Verfügung stehende Kapazität in Absprache mit den Beteiligten. Er ist ebenfalls verantwortlich für die Disposition von allenfalls verschobenen Wahloperationen. Er ist somit eine Art Drehscheibe für das Tagesgeschäft mit dem Ziel, die zur Verfügung stehende OPS-Kapazität optimal zu nutzen. 


\section{Abbildung 2}

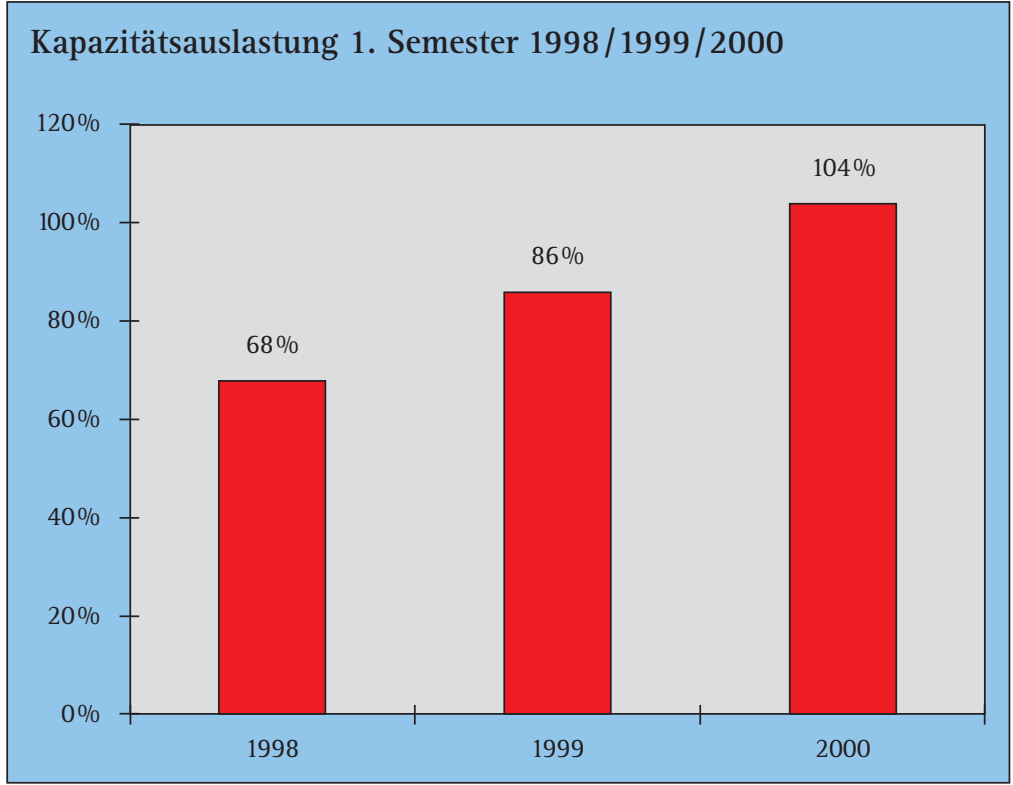

Pünktlichkeit Vergleich 1. Semester 1998/1999/2000

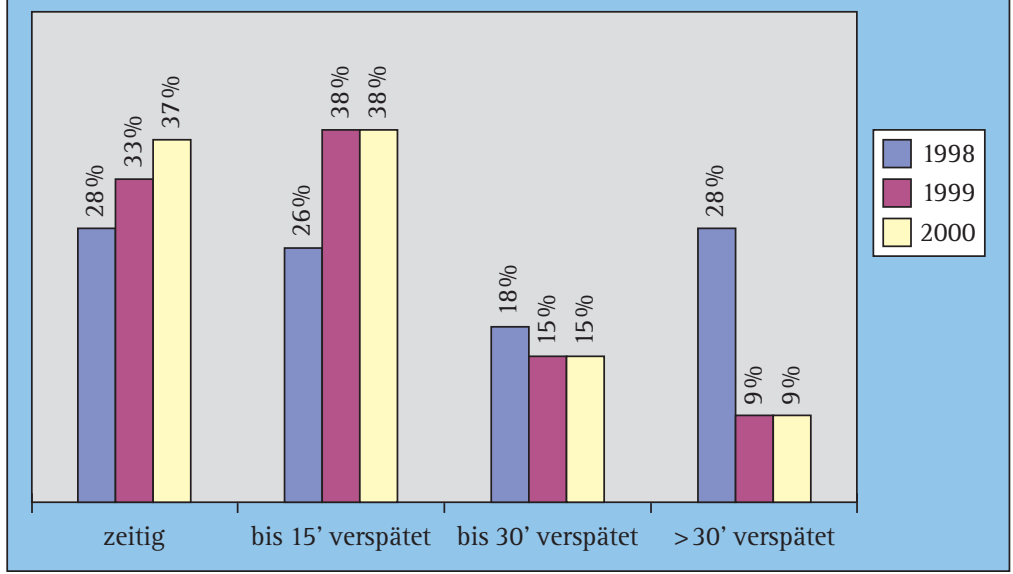

Start zur Neuorganisation

Nach vielen Sitzungen und Gesprächen wurde am 1. Januar 1999 diese neue OPS-Organisation eingeführt. Dabei konnten wir für die Zuteilung der Saalkapazität auf ein bereits $1 \frac{11}{2}$ Jahre zuvor eingeführtes Controlling zurückgreifen.

\section{OPS-Controlling}

Im OPS-Controlling werden die exakten Daten aller Operationen erfasst. Diese werden dann verglichen mit den zugeteilten Operationskapazitäten sowie den geplanten Operationszeiten. Daraus ergeben sich Auswertungen über:
- Anzahl Operationen;

- Anzahl Operationsstunden im Elektivprogramm und im Notfallprogramm;

- Auslastung der Kapazität pro Klinik;

- Pünktlichkeit des Operationsbeginns am Morgen;

- Wechselzeiten;

- unproduktive Zeit (Verzögerung am Morgen, Wechselzeiten >45 Minuten, sowie ungenutzte OP-Kapazität);

- Auslastung der Notfallsäle in bezug auf die Tageszeit.

Die Auswertungen werden in einem Wochen- und in einem Monatsbericht den Entscheidungsträgern zugestellt. Ebenfalls werden eine Jahreszusammenfassung sowie Vergleiche zwischen den einzelnen Jahren angestellt.

\section{Bessere Auslastung erhöht den Druck}

Durch diese Massnahmen konnten 1999 die Auslastung der Operationssäle sowie die Produktivität (erwirtschaftete Taxpunkte pro Mitarbeiter) deutlich verbessert werden. Die folgende Grafik zeigt die Auslastung der elektiven Operationskapazität im Jahr vor der neuen OP-Organisation (1998) sowie in den beiden ersten Jahren. Es ist uns dabei gelungen, die Saalauslastung und den pünktlichen Operationsbeginn am Morgen zu optimieren (Abb. 2).

Allerdings hatte diese Verbesserung der wirtschaftlichen Seite auch negative Auswirkungen. Der Druck auf den einzelnen Mitarbeiter nahm deutlich zu. Durch den bereits vorhandenen Mangel an Instrumentierschwestern wurde dieser noch einmal verstärkt. Im weiteren waren unsere durch kantonale Vorgaben diktierten Löhne nicht marktgerecht. Diese Faktoren führten zu Unzufriedenheit.

\section{Zufriedenheit als Massstab}

Es war mir daher ein Anliegen, im zweiten Jahr der Neuorganisation die Qualität des Arbeitsplatzes und die Zufriedenheit des Personals zur Hauptzielsetzung zu erklären. Für diese nicht ganz einfache Aufgabe habe ich die professionelle Hilfe einer auf Kommunikation spezialisierten Firma (FMH Marketing + Kommunikation) in Anspruch genommen: Das Projekt TOPS wurde vorbereitet und im Januar 2000 mit der unvergesslichen OPS-Beach-Party im Hallenbad und der eine Woche später durchgeführten Präsentation des Projektes gestartet. Zielsetzung war die Verbesserung des Arbeitsklimas durch ein Training zur Konfliktbewältigung und durch eine Verbesserung der internen und externen Kommunikation. Zudem wurde parallel zu diesem Projekt in Zusammenarbeit mit dem Institut für medizinische Ökonomie des Universitätsspitals Zürich eine Umfrage zur Erfassung der Mitarbeiterzufriedenheit im Operationssaal durchgeführt. 


\section{Auf neuen Wegen}

Das Projekt hat verschiedene Teilbereiche. Ein Hauptbereich ist die sogenannte $360^{\circ}$-Beurteilung von neun "Change-Agents». Dabei werden neun "Schlüsselpersonen" aus allen Personengruppen von je insgesamt sechs Mitarbeitern (Vorgesetzte, Kollegen und Untergebene) nach einem vorgegebenen Fragebogen beurteilt. Beurteiler und Schlüsselpersonen wurden vorgängig mündlich durch das TOPS-Berater-Team informiert und instruiert. Die neun Schlüsselpersonen erhielten dann in einem zweitägigen Seminar Ende Mai das Feedback dieser Beurteilungen sowie ein Konflikt- und Kommunikationstraining. Von ihnen aus soll dann eine Veränderung (deshalb ChangeAgents) ausgehen.

\section{Kommunikation als Schlüssel zur Veränderung}

Ein weiterer Teil ist die Kommunikation. Wir haben mit den monatlich erscheinenden TOPS-Team-News, einem Informationsblatt für alle OPS-Mitarbeitenden, eine Plattform geschaffen, welche es ermöglichen soll, $\mathrm{zu}$ informieren und $\mathrm{zu}$ diskutieren. Wir möchten damit auch Transparenz schaffen um so die Motivation, gute Leistungen $\mathrm{zu}$ erbringen, zu erhöhen.

\section{Wo liegen unsere Hauptprobleme}

Aufgrund der Resultate der oben erwähnten Mitarbeiterzufriedenheitsumfrage sind Hektik, zu tiefer Lohn, überladene Operationsprogramme und fehlende Zeit für die Patienten die Problempunkte, die am meisten Unzufriedenheit verursachen.

Wir haben bereits einiges unternommen, um in dieser Richtung Verbesserungen $\mathrm{zu}$ erreichen. So wurden in Sachen Lohn deutliche Fortschritte erzielt. Um Hektik abzubauen, wurde nun auch bei den Instrumentierschwestern der 3-Schichten-Betrieb eingeführt, so dass überlange Präsenz- und Arbeitszeiten der Vergangenheit angehören.

Es wurde im weiteren ein Briefkasten aufgestellt, wo alle Mitarbeiterinnen und Mitarbeiter die Möglichkeit haben, Verbesserungsvorschläge anzubringen und Probleme anzusprechen. Diese werden von den Change-Agents, welche sich in regelmässigen Zusammenkünften treffen, bearbeitet. Wir möchten damit versuchen, die Anliegen jedes Mitarbeiters und jeder Mitarbeiterin ernst zu nehmen und diesen auch eine unbürokratische Möglichkeit geben, Einfluss zu nehmen.
Als weiterer Schritt wurde auch aufgrund der neuesten Controllingdaten mit den nach Kliniken aufgeschlüsselten Kapazitätsauslastungen die Saalzuteilung neu angepasst. Wir hoffen so, die Kapazität noch gleichmässiger nutzen zu können, um damit Hektik und das "Überziehen» von Operationsprogrammen abzubauen. Ebenso werden die Klinikchefs regelmässig über die Situation orientiert und darauf hingewiesen, die Planung der Operationen der vorhandenen Kapazität realistisch anzupassen.

\section{Wo liegen unsere Stärken?}

Die im Mai 2000 durchgeführte Mitarbeiterzufriedenheitsumfrage hat neben den oben erwähnten Problempunkten auch eine ganze Reihe von sehr erfreulichen Resultaten ergeben. So sind über 90\% der Mitarbeiterinnen und Mitarbeiter, welche geantwortet haben, so zufrieden, dass sie am Morgen gerne in den OPS arbeiten kommen. Ebenso finden über 90\% der Mitarbeiterinnen und Mitarbeiter die Arbeit interessant, abwechslungsreich und verantwortungsvoll. Knapp 70\% sind der Meinung, dass ihre Arbeit von den Vorgesetzten geschätzt wird.

\section{Beurteilung}

Ich bin überzeugt, dass wir mit unserer OPS-Neuorganisation einen für ein öffentliches Spital neuen und zukunftsweisenden Weg gegangen sind. Er hat uns ermöglicht, unsere knappen Ressourcen optimal auszunutzen. Die Kehrseite der Medaille ist der erhöhte Druck auf die Mitarbeiter, der durch die Optimierungsmassnahmen ausgeübt wurde. Wir haben auch diese Seite ernst genommen und einen Teil der Einsparungen im Projekt TOPS den Mitarbeitern wieder zugute kommen lassen und so in die Zukunft unseres Arbeitsplatzes investiert. Gerade in einem so komplexen Betrieb wie die Operationsabteilung mit einer Vielzahl von Schnittstellen, Berufsgruppen, Interessen und Hierarchiestufen kommt der Kommunikation und der Art und Weise, wie Probleme und Konflikte angegangen werden, eine entscheidende Bedeutung zu. Neben den Bemühungen der OPS-Leitung liegt es jedoch an allen, dazu beizutragen, dass der OPS ein Arbeitsplatz ist, wo es Freude macht mitzuarbeiten. Let's build a truly great OPS - together! 